\title{
UN CASO RARO DE FIBROMIXOMA ODONTOGÉNICO CON APARIENCIA RADIOGRÁFICA DE “RAYOS DE SOL". REPORTE DE CASO Y REVISIÓN DE LA LITERATURA
}

\author{
A RARE RADIOGRAPHIC "SUNRAY" APPEARANCE IN ODONTOGENIC FIBRO-MYXOMA. CASE REPORT AND \\ REVIEW OF THE LITERATURE
}

\author{
Cecilia Paniagua Chacón * \\ Víctor Calderón Ubaqui ** \\ Jorge Beltrán Silva ***
}

\section{RESUMEN:}

Se presenta un caso de Fibromixoma Odontogénico (FO) con aspecto radiológico de "rayos de sol" el cual es poco frecuente en este tipo de lesión y entre las lesiones benignas; sin embargo, en la revisión de la literatura han sido reportados casos similares. El objetivo de esta presentación es mostrar este aspecto que se puede mimetizar con una lesión significativa como es el sarcoma osteogénico y que puede llevar al profesional en Estomatología e incluso al especialista en Radiología Oral a un error en el diagnóstico.

PALABRAS-LLAVE: Fibromixoma odontogénico - Rayos de sol.

\section{ABSTRACT:)}

A case of Odontogenic Fibro-myxoma (FO) with radiographic appearance of "sunrays" is presented which is slightly frequent in this type of injury and among the benign injuries; nevertheless, in the review of the literature similar cases have been reported. The aim of this presentation is to show this aspect mimicking a significant pathology since it is the osteogenic sarcoma and can lead the professional in Stomatology and even to the specialist in Oral Radiology to a mistake in the diagnosis.

DESCRIPTORS: Odontogenic Fibro-myxoma - Sunrays appearance.

\footnotetext{
* Especialista en Radiología Oral y Máxilofacial

** Especialista en Radiología Oral y Máxilofacial Profesor Asociado del Departamento Académico Medicina, Cirugía y Patología Oral Facultad de Estomatología Universidad Peruana Cayetano Heredia

*** Especialista en Radiología Oral y Maxilofacial Magíster en Educación Superior Profesor Asociado del Departamento Académico Medicina, Cirugía y Patología Oral Facultad de Estomatología Universidad Peruana Cayetano Heredia
} 
Paniagua Chacón C, Calderón Ubaqui V, Beltrán Silva J. Un caso raro de fibromixoma odontogénico con apariencia radiográfica de "rayos de sol". Reporte de caso y revisión de la literatura. Revista de Odontologia da Universidade Cidade de São Paulo 2008 jan-abr; 20(1):93-9

\section{INTRODUCCION}

El mixoma odontogénico (MO) es una neoplasia intraósea. (Barnes et al. ${ }^{2}$, 2005) La frecuencia del MO varia en diferentes partes del mundo entre el 3 - $20 \%$ de todos los tumores odontogénicos. En la mayoría de estudios el $\mathrm{MO}$ es el tercer tumor odontogénico mas frecuente seguido del odontoma y ameloblastoma. (Adekeye et al. ${ }^{1}$, 1984; Cuestas-Carneiro ${ }^{4}$, 1988) presentándose mayormente en mujeres entre la segunda y cuarta década. (Barnes et al. ${ }^{2}$, 2005) Dos tercios del MO son localizados en la región molar de la mandíbula (Adekeye et al. ${ }^{1}$, 1984; Cuestas-Carneiro $\left.{ }^{4}, 1988\right)$. La perdida dentaria resultante de efectos de un $\mathrm{MO}$ es rara pudiendo estar asociada a reabsorción, desplazamiento y perdida dental. (Adekeye et al. ${ }^{1}$, 1984; Cuestas-Carneiro $\left.{ }^{4}, 1988\right)$.

Las características radiológicas del MO descritas en la literatura son conflictivas. (Farman et al. $\left.{ }^{6}, 1993\right)$ Se puede presentar como una imagen radiolúcida/radiopaca, unilocular, multilocular o peri coronal de límites poco definidos y bordes corticados. La presencia de septos es característica del MO los cuales producen la apariencia radiográfica de los patrones de "pompas de jabón", "panal de abejas", "rayos de sol" y "raqueta de tenis", este último es el más patognomónico. (Schimidseder $e t$ al. ${ }^{16}$; Peltola et al. ${ }^{15}$, 1994).

La literatura disponible refiere que el $\mathrm{MO}$ es de carácter odontogénico debido a que podría originarse del mesénquima dental o del ligamento periodontal. (Halspenny et al. ${ }^{7}, 2000$ ).

Se presenta un caso de $\mathrm{MO}$ con aspecto radiológico de "rayos de sol" el cual es poco frecuente en este tipo de lesión.

\section{REPORTE DE CASO}

Una mujer de 17 años se presentó al servicio de Cirugía Oral y Maxilofacial del Hospital Nacional Cayetano Heredia refiriendo que hace aproximadamente 2 años notó un aumento de volumen en la región geniana baja del lado izquierdo. Posteriormente presentó movilidad dentaria con avulsión espontánea de la premolar inferior izquierda. Hace 8 meses, sintió adormecimiento del hemilabio inferior izquierdo por lo que acudió a un puesto de salud de su localidad donde recibió tratamiento con antibióticos sin presentar mejoría alguna. En estas condiciones fue referida a nuestro servicio.

A al evaluación clínica se observó aumento de volumen en la región geniana baja izquierda causado por

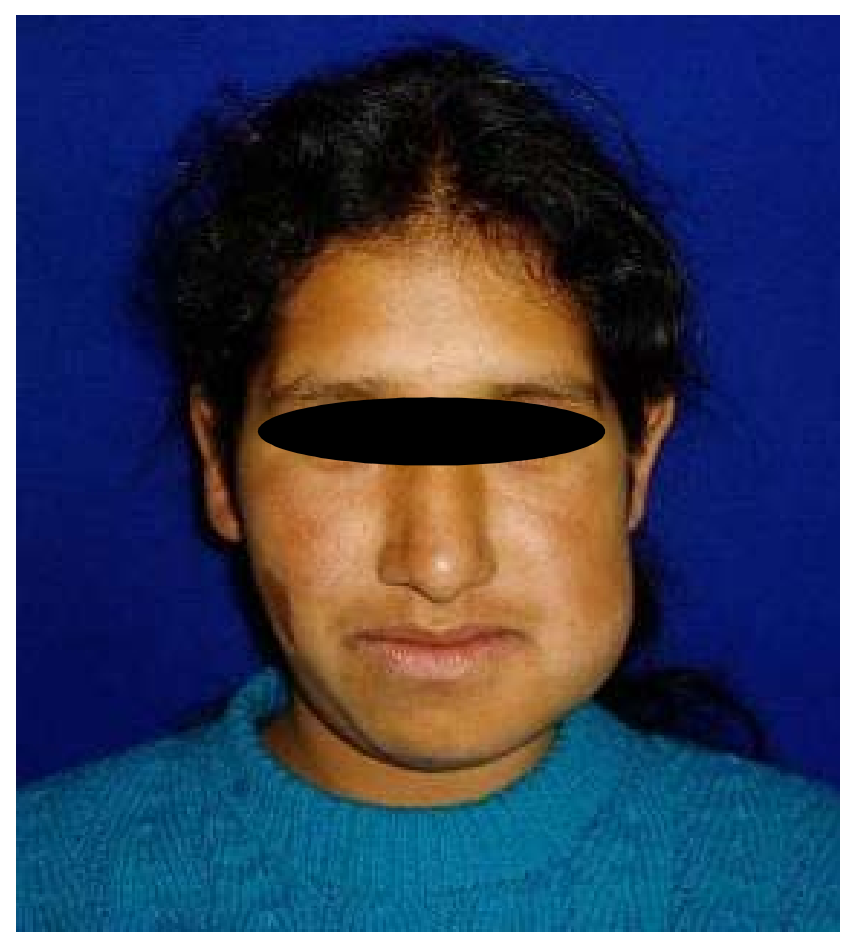

Fig. 1 Aumento de volumen en región geniana izquierda

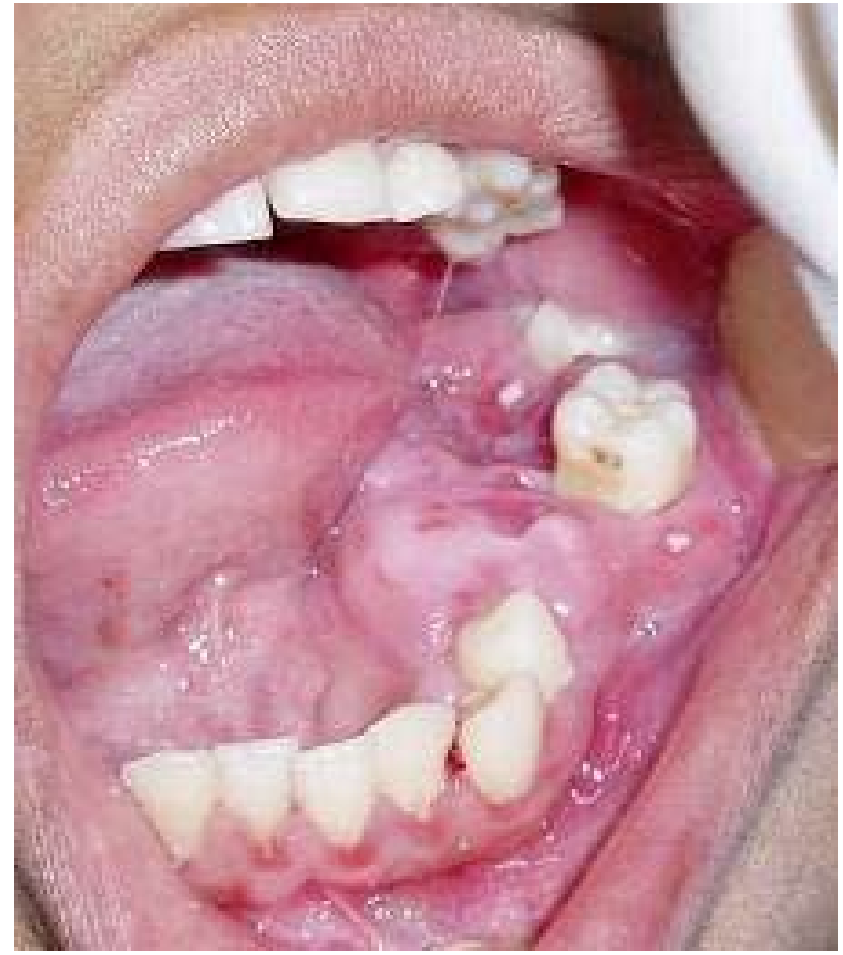

Fig. 2 Tumor con indentaciones oclusales antagonistas

efecto de masa (Fig. 1) de un tumor intraoral de consistencia firme a la palpación con un tamaño aproximado de $5 \times 5 \times 2 \mathrm{~cm}$. Se pudo observar solución de continuidad 
Paniagua Chacón C, Calderón Ubaqui V, Beltrán Silva J. Un caso raro de fibromixoma odontogénico con apariencia radiográfica de "rayos de sol". Reporte de caso y revisión de la literatura. Revista de Odontologia da Universidade Cidade de São Paulo 2008 jan-abr; 20(1):93-9

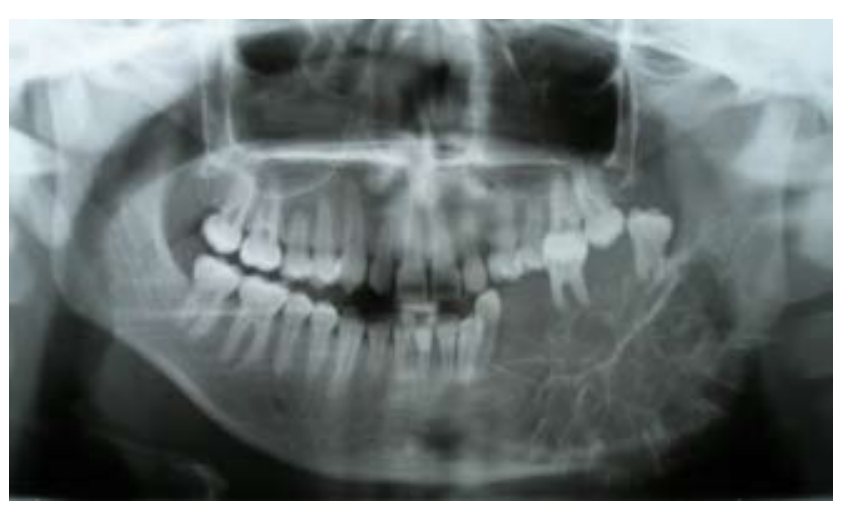

Fig. 3 Radiografía Panorámica con aspecto de "rayos de Sol" y "diente flotante"

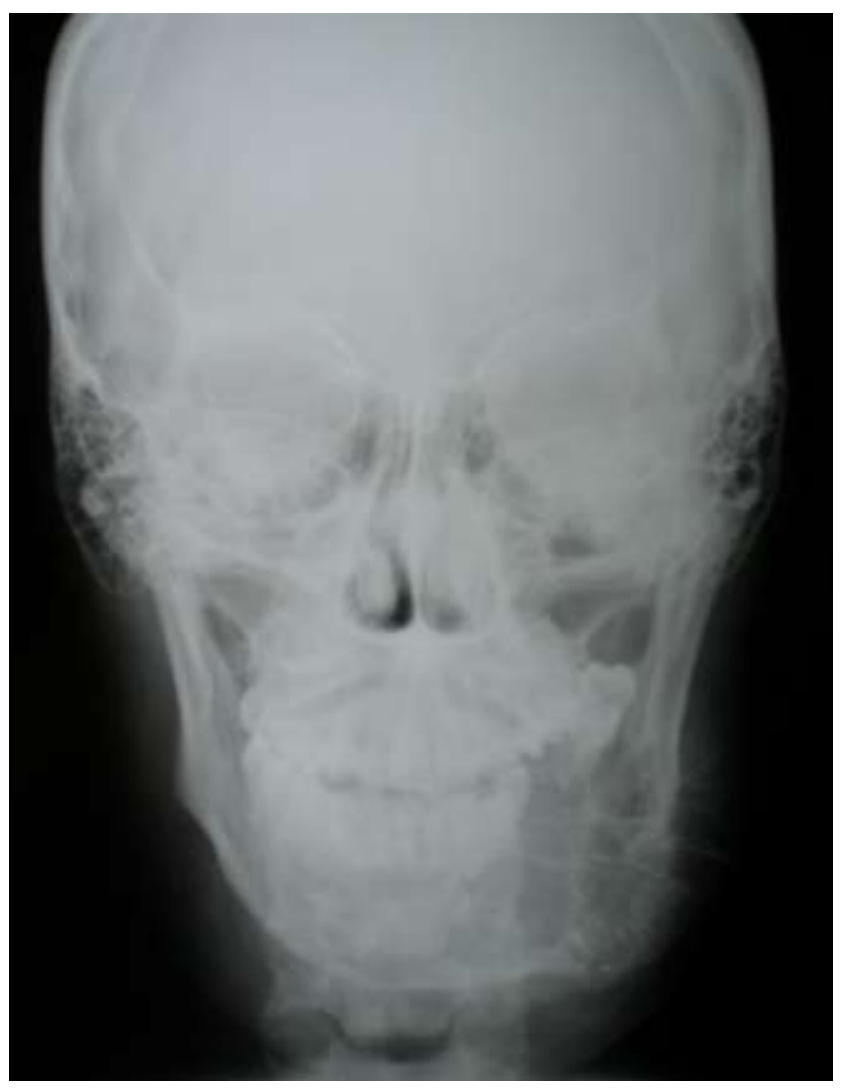

Fig. 4 Radiografia Postero Anterior con aspecto de "rayos de sol" $y$ "diente flotante"

en la superficie de la lesión debido a las marcas de las piezas dentarias antagonistas. No se palparon adenopatías. Las piezas dentarias 3.4, 3.6 y 3.8 presentaron movilidad (Fig.2). No refirió dolor a la palpación.

La radiografía panorámica mostró una imagen radiolúcida multilocular ubicada en parasínfisis, cuerpo, ángulo y tercio inferior de la rama ascendente mandibular izquierda, de límites definidos y bordes parcialmente

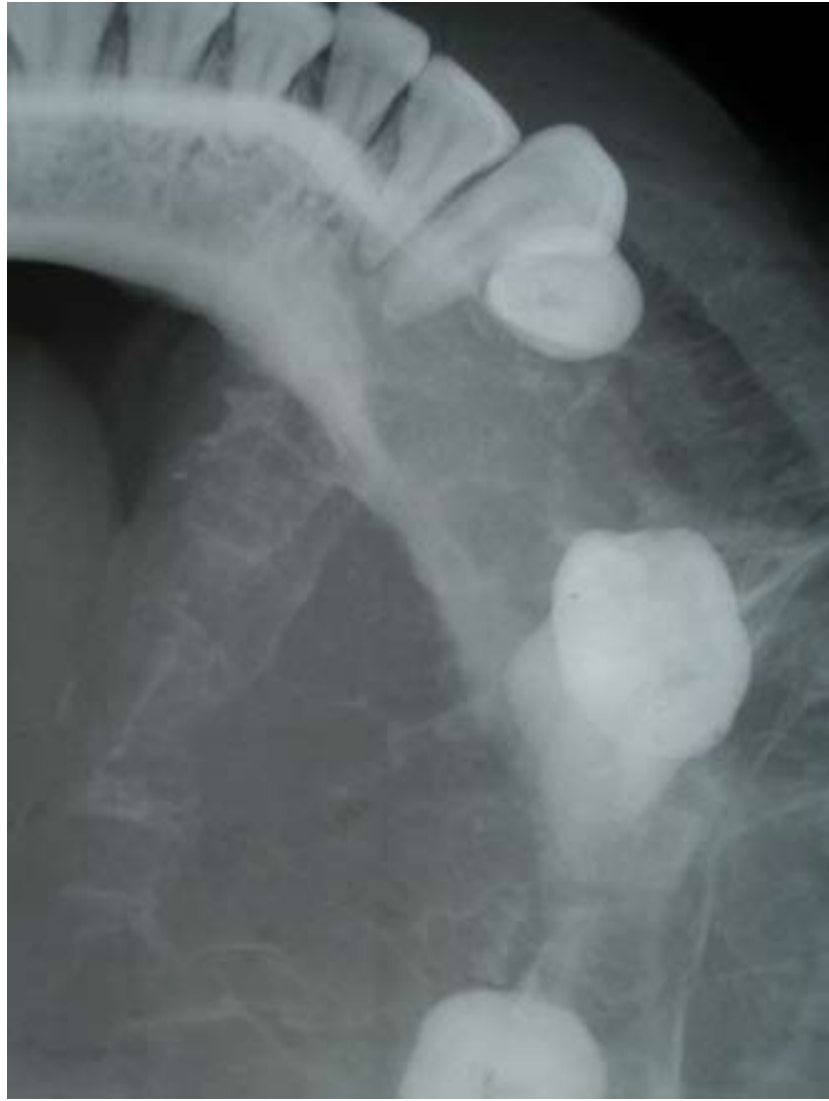

Fig. 5 Radiografía Oclusal con tablas óseas expandidas adelgazadas y discontinuas

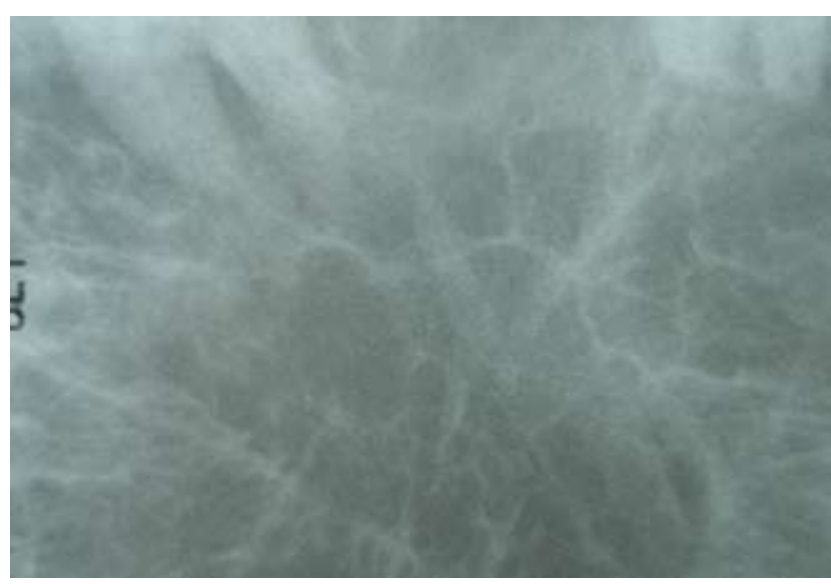

Fig. 6 Radiografia Periapical con aspecto de "raqueta de tenis"

corticalizados con aspecto de "rayos de sol". El conducto dentario inferior se mostró desplazado en dirección lateral y caudal. Las piezas dentarias se mostraron extruídas sin lámina dura dando un aspecto de "diente flotante" (Fig. 3).

La radiografía póstero anterior mostró expansión de 
Paniagua Chacón C, Calderón Ubaqui V, Beltrán Silva J. Un caso raro de fibromixoma odontogénico con apariencia radiográfica de "rayos de sol". Reporte de caso y revisión de la literatura. Revista de Odontologia da Universidade Cidade de São Paulo 2008 jan-abr; 20(1):93-9

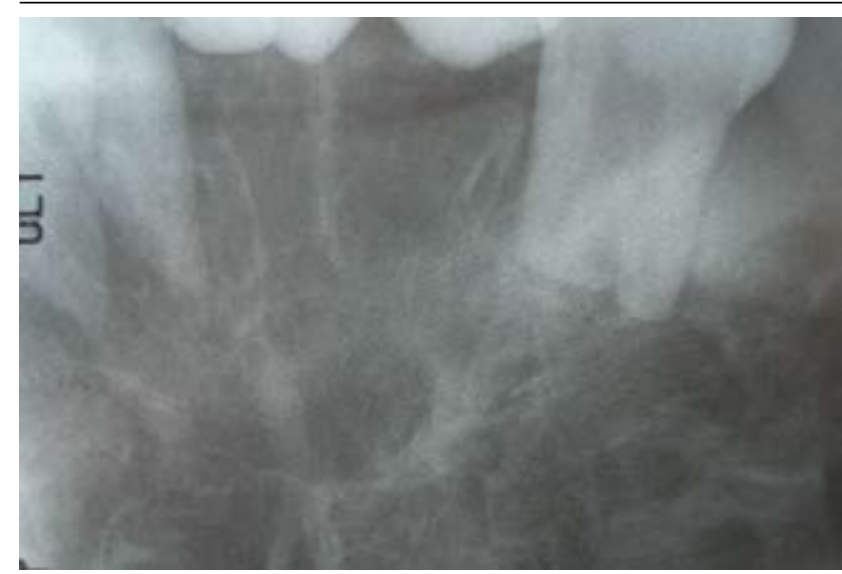

Fig. 7 Radiografía Periapical con ensanchamiento del espacio para el ligamento periodontal y presencia de lámina dura.

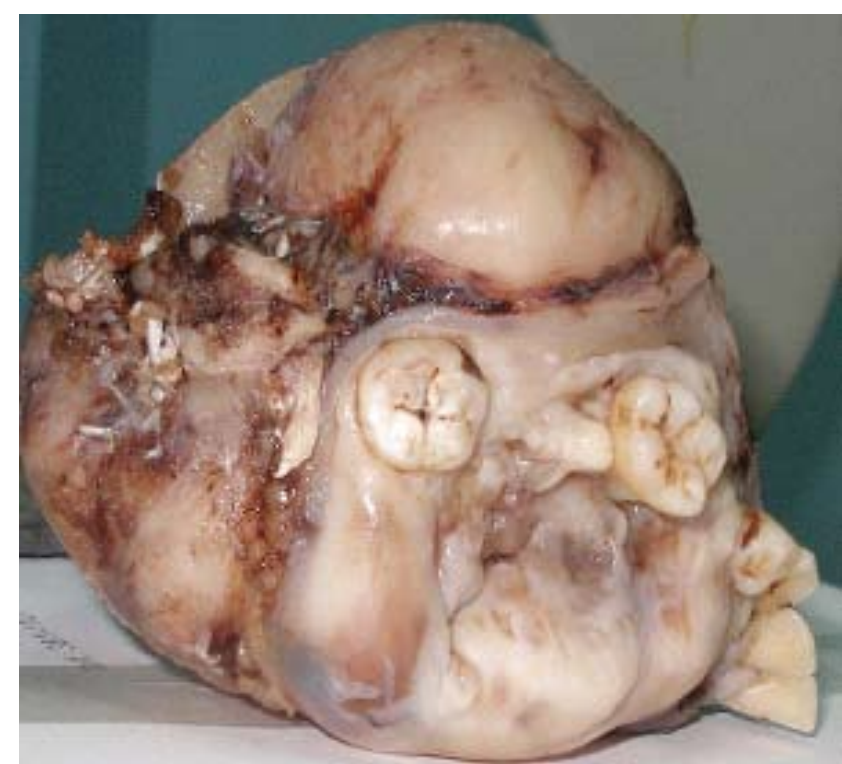

Fig. 8 Fotografía del tumor excisionado (espécimen)

ambas tablas óseas con aspecto de "rayos de sol". Las piezas dentarias mostraron también aspecto de "diente flotante" (Fig. 4).

La radiografía oclusal mostró las corticales lingual y vestibular discontinuas, expandidas y adelgazadas así como también la presencia de espículas óseas (Fig.5).

Las radiografías periapicales mostraron finos tabiques entrecruzados en ángulo recto con aspecto de "raqueta de tenis". Las piezas dentarias 3.8, 3.6 y 3.4 se mostraron desplazadas en dirección cefálica con ensanchamiento del espacio para el ligamento periodontal y presencia de lámina dura, no se observó reabsorción radicular (Figs. 6 y 7$)$.

Entre los diagnósticos presuntivos se consideró una lesión tumoral benigna; sin embargo, las radiografías

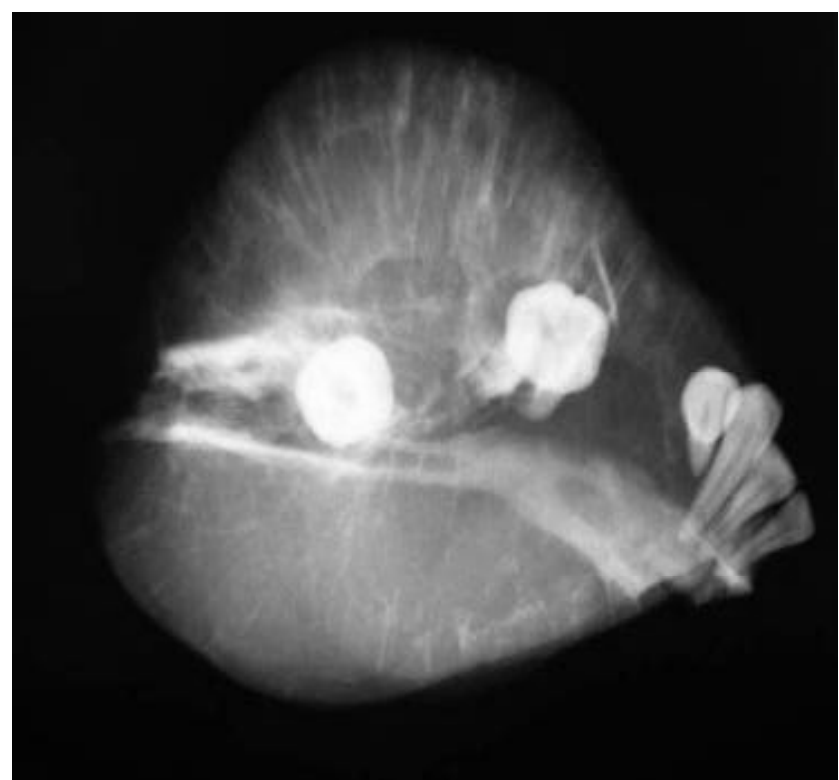

Fig. 9 Radiografía del tumor excisionado con aspecto del "rayos de sol"

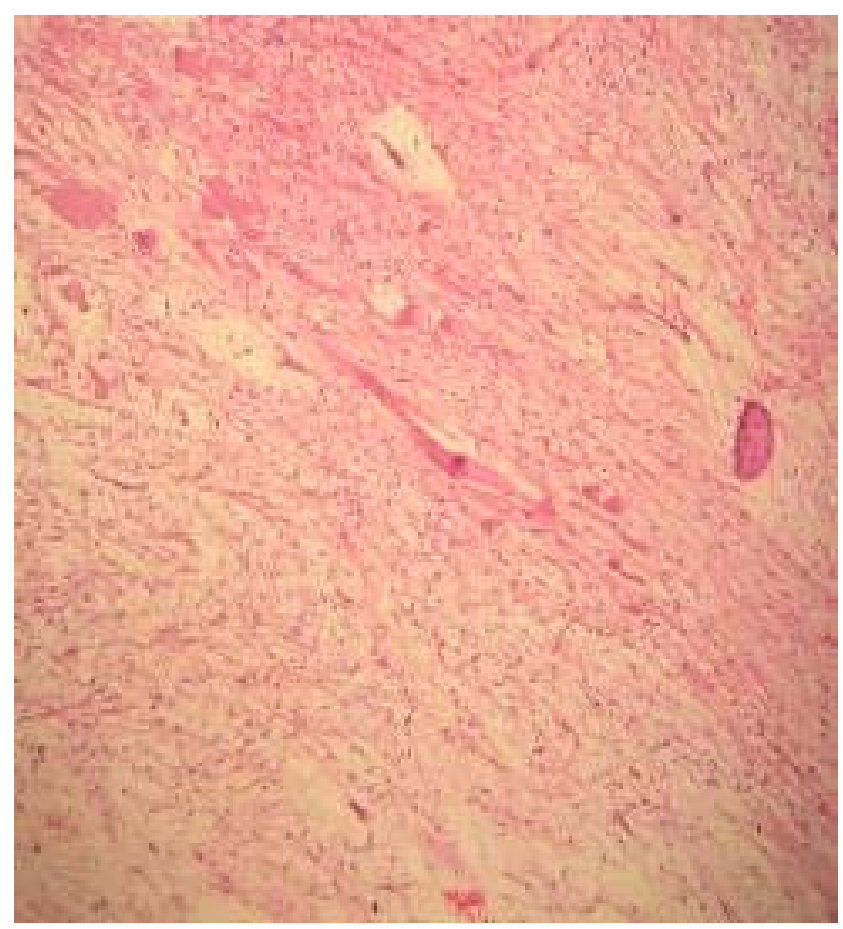

Fig. 10 Células fusiformes y de forma estrellada en matriz mixomatosa con presencia de fibras colágenas y trabéculas de bueso compatible con Fibromixoma

revelaron características radiográficas tanto de lesión maligna como de benigna lo cual llevó a considerar al osteosarcoma como diagnóstico diferencial.

La biopsia incisional del tumor mostró presencia de 
Paniagua Chacón C, Calderón Ubaqui V, Beltrán Silva J. Un caso raro de fibromixoma odontogénico con apariencia radiográfica de "rayos de sol". Reporte de caso y revisión de la literatura. Revista de Odontologia da Universidade Cidade de São Paulo 2008 jan-abr; 20(1):93-9

tejido mixomatoso dispuesto en compartimientos separados por trabéculas óseas. No se distinguieron cambios atípicos. El diagnóstico anatomopatológico preoperatorio fue Mixoma. Se planeó la resección en bloque del tumor seguida de la colocación de una placa de reconstrucción e injerto córtico-esponjoso de la cresta ilíaca (Figs. 8 y 9).

El estudio anatomopatológico de la pieza quirúrgica mostró células fusiformes y células de forma estrellada dentro de una matriz mixomatosa que en algunas áreas cambiaban por la presencia de fibras colágenas, también se distinguieron trabéculas de hueso que producían compartimientos dentro del tejido descrito, siendo su diagnóstico definitivo Fibromixoma (Fig. 10)

\section{DISCUSION}

El MO, ocasionalmente llamado mixofibroma, es un tumor raro, pero importante de los maxilares. A pesar de ser benigno, muestra frecuentemente un carácter agresivo de infiltración a los tejidos blandos y tendencia a recurrir después de la cirugía. (Barnes et al. ${ }^{2}, 2005$ )

En 1863, Virchow usó el termino mixoma para un grupo de tumores que tenían semejanza histológica con la sustancia mucinosa del cordón umbilical. En 1948 Stoud redefinió los criterios histopatológicos existentes describiendo a los mixomas como neoplasias verdaderas que no metastatizan, excluyendo la presencia de componentes celulares reconocibles de otros tejidos mesenquimales como los condroblastos, lipoblastos y rabdomioblastos. (Buchner et al. ${ }^{3}, 2006$ )

El 16\% de los tumores odontogénicos corresponden al mixoma odontogénico sin diferencia significativa del género, sin embargo otros reportes mencionan una predilección por el sexo femenino. (Simon et al. ${ }^{17}, 2004$; Barnes et al. ${ }^{2}$, 2005) La lesión puede estar asociada a dientes no erupcionados o a regiones con agenesia dentaria. (Halfpenny et al. ${ }^{7}, 2000$ )

Es de crecimiento lento consistente en la acumulación de sustancia mucoide con algo de colágeno (MacDonald-Jankowski et al. $\left.{ }^{11}, 2004\right)$. Cuando se evidencia una relativa cantidad de colágeno se puede usar el término de mixofibroma (Barnes et al. ${ }^{2}$, 2005). Se caracteriza por la presencia de células estrelladas y fusiformes embebidas en una abundante matriz extracelular mixoide y mucoide. Ocasionalmente el MO muestra la presencia de epitelio odontogénico relacionado al mesénquima de un germen dentario o al ligamento periodontal. Originándose raras veces en otras partes del esqueleto (MacDonald-Janko- wski et al. ${ }^{11}$, 2004).

Existe una predilección significativa para la localización del tumor: Dos tercios en la mandíbula y un tercio en el maxilar superior. La región posterior es la más frecuentemente afectada (67\%) (Olgac et al. ${ }^{14}$, 2006; Barnes et $a .^{2}$, 2005). Los MO de mayor tamaño causan expansión indolora y perforación de la cortical mostrando su naturaleza expansiva. (Farman et al. ${ }^{6}$, 1993)

Los términos aplicados a la definición de márgenes de la lesión son los ampliamente descritos por White y Pharoah catalogados de la siguiente manera: bien definido, el cual puede presentar una cortical, y pobremente definido (MacDonald-Jankowski et al. ${ }^{12}$, 2002). El grado de definición establecido por Slootweg y Muller considera a los MO como bien definidos cuando su radiodensidad cambia marcadamente a una distancia de 1 $\mathrm{mm}$ en la interfase entre la lesión y el hueso circundante (Slootweg, Mueller ${ }^{18}$, 1990). Los bordes del MO suelen producir problemas a la hora de la descripción (Olgac et al. $\left.{ }^{14}, 2006\right)$ por lo que puede llevar a confusión en el diagnóstico, dichas formas pueden ser unilocular o multilocular siendo esta última la presentación más común (MacDonald-Jankowski et al. ${ }^{12}$, 2002; Zhang et al. ${ }^{20}$, 2007).

Los MO de gran tamaño pueden presentar desplazamiento radicular así como reabsorción radicular y reacción periostal.

Los patrones radiográficos creados por los septos son en la mayoría de veces de "pompas de jabón”, "panal de abejas" y "raqueta de tenis".

La apariencia radiográfica del MO ocasionalmente agresiva, es raramente sugestiva de malignidad. De Labrouhe $^{5}, 1994$, reportó un caso en maxilar representado por una lesion destructiva acompañada de una apariencia en "rayos de sol". Mlosek ${ }^{13}$ reportó dos casos de mixosarcomas los cuales resultaron en muerte dos años después de operados. Peltola et al. ${ }^{15}$, Schimidsedr et al. ${ }^{16}, 1978$; reportaron dos lesiones con una apariencia de "rayos de sol" usualmente sugestiva de sarcoma.

Zhang et al. $.^{20}, 2007$ estudiaron 41 casos de MO los cuales fueron clasificados en seis grupos según las características radiográficas: Tipo: I: unilocular, Tipo II: multilocular, Tipo III: con compromiso del reborde alveolar, Tipo IV: con compromiso del seno maxilar, Tipo V: destrucción osteolítica y Tipo VI: una combinación de destrucción osteolítica con osteogénesis. El aspecto radiográfico de "rayos de sol" fue catalogado dentro del grupo VI. Solamente dos pacientes presentaron dicha 
Paniagua Chacón C, Calderón Ubaqui V, Beltrán Silva J. Un caso raro de fibromixoma odontogénico con apariencia radiográfica de "rayos de sol". Reporte de caso y revisión de la literatura. Revista de Odontologia da Universidade Cidade de São Paulo 2008 jan-abr; 20(1):93-9

característica. Llegaron a la conclusión que si el tumor perfora la cortical con formación de espículas radiadas múltiples, la apariencia radiográfica será de "rayos de sol". Esta apariencia es común al osteosarcoma, no obstante, debe existir integración entre los síntomas clínicos, la duración de la enfermedad y la velocidad de crecimiento del tumor (Zhang et al. ${ }^{20}, 2007$ ). Para Farman et al. ${ }^{6}, 1993$, la apariencia mixta puede deberse al hueso residual y no a neo formación ósea.

El diagnóstico radiográfico diferencial incluye lesiones que muestran radiolucencias multiloculares típicas como el ameloblastoma, hemangioma central, quiste óseo aneurismático, granuloma central de células gigantes, querubismo y tumores metastásicos de los maxilares, etc. (Kim et al. ${ }^{9}, 2002$ )

La parestesia puede estar asociada a lesiones mandibulares a pesar de su rareza. (Adekeye et al. ${ }^{1}$, 1984; Cuestas Carnero ${ }^{4}$, 1988)

El tratamiento recomendado para el $\mathrm{MO}$ es la ciru- gía radical o la excisión conservadora dependiendo del tamaño del tumor. La resección radical incluye márgenes de 1.5 a $2 \mathrm{~cm}$ de hueso sano (Halfpenny et al. ${ }^{7}, 2000$ ).

El pronóstico de los $\mathrm{MO}$ es por lo general bueno, a pesar que se ha descrito recurrencia después de 30 años de la cirugía.20 Los pacientes deben someterse a un seguimiento cercano dentro de los siguientes dos años por ser el tiempo más probable para la recurrencia. La remoción incompleta es el factor causal principal para la recurrencia. (Landa et al. ${ }^{10}, 2002$; Halfpenny et $\mathrm{al}^{7}$, 2000).

\section{CONCLUSION}

Para concluir, no obstante la apariencia radiográfica rara, se debe integrar el aspecto de "rayos de sol" a las características radiográficas del $\mathrm{MO}$ además de hacer un análisis de la historia clínica y los hallazgos físicos, especialmente cuando se intenta diagnosticar lesiones sin las características frecuentes. 
Paniagua Chacón C, Calderón Ubaqui V, Beltrán Silva J. Un caso raro de fibromixoma odontogénico con apariencia radiográfica de "rayos de sol". Reporte de caso y revisión de la literatura. Revista de Odontologia da Universidade Cidade de São Paulo 2008 jan-abr; 20(1):93-9

\section{REFERENCIAS}

1. Adekeye EO, Abery ES, Edwards MB, Williams HK. Advanced central mixoma of the jaws in Nigeria. Clinical features, treatment and pathogenesis. Intern J Oral Surg 1984 Jun;13(3):177-86.

2. Barnes L, Eveson JW, Reichart PA, Sidransky D eds. Pathology and genetics of head and neck tumours. Lyon: IARC Press, 2005, cap 6.

3. Buchner A, Merrel PM, Carpenter WM. Relative frequency of central odontogenic tumors: a study of 1,088 cases from Northern California and comparison to studies from other parts of the world. $J$ Oral Maxillofac Surg 2006 Sep; 64(9):1343-52.

4. Cuestas-Carnero R. Odontogenic mixoma: Report of a case. J Oral Maxillofac Surg 1988; 46: 705-9

5. De Labrouhe C, Bertand JC, Guilbert F. Radiologic aspects of myxomas of the jaws. A series of $27 \mathrm{ca}-$ ses. Rev Stomatol Chir Maxillofac 1994; 95(2): 80-3.

6. Farman AJ, Nortjé CJ, Wood LE. Oral and maxillofacial diagnostic imaging. ST Louis: CV Mosby 1993. pp 257-260

7. Halfpenny W. Verey A, Bardsley V. Myxoma of the mandibular condyle a case report and review of the literature. Oral Surg, Oral Med Oral Pathol Oral Radiol, Endod 2000 Sep; 90(3): 348-53.

8. Kaffé I, Naor H, Buschner A. Clinical and radiological features of odontogenic myxoma of the jaws. Dentomaxillofac Radiol 1997 Sep; 26(5): 299-303.

9. Kim JY, Park GM, Cho BH, Nah KS. Odontogenic myxoma report of 2 cases. Korean J Oral Maxillofac Radiol 2002; 32: 231-4.

10. Landa LF, Hedrick MH, Nepomuceno-Perez MC, Sotereanos G. Recurrent myxoma of the zygoma: a case report. J Oral Maxillofac Surg 2002 Jun; 60(6): 704-8.
11. McDonald Jankowski DS, Yeung WK, Li T, Lee KM. Computed tomography of odontogenic myxoma. Clinical Radiology 2004 Mar; 59(3): 281-87.

12. McDonald Jankowski DS, Yeung WK, Li T, Lee KM. Odontogenic myxoma in the Hong Kong Chinese: Clinico radiological presentation and systematic review. Dentomaxillofac Radiol 2002 Mar; 31(2): 71-83.

13. Mlosek K, Kryst L, Piekarczyk J. Odontogenic myxomas. Pol Przegl Radiol Med Nukl 1982 Jan-Jun; 46(1-3): 21-6.

14. Olgac V, Koseoglu BG, Aksakall N. Odontogenic tumours in Istanbul: 527 cases. Br J Oral and Maxillofac Surg 2006 Oct; 44(5): 386-8.

15. Peltola J, Magnusson B, Happonen RP, Borrman H. Odontogenic myxoma-and radiographic study of 21 tumours. Br J Oral Maxillofac Surg 1994 Oct; 32(5): 298-302

16. Schmidseder R, Groddeck A, Scheunemann H. Diagnostic and therapeutic problem of mixoma (myxofibroma) of the jaws. Journal Maxillofacial Surgery 1978 Nov 6(4): 281-6.

17. Simon EN, Merkx MA, Vuhahula E, Ngassapa D, Stoelinga PJ. Odontogenic myxoma: a clinicopathological study of 33 cases. Int. J. Oral Maxillofac. Surg. 2004 Jun; 33(4): 333-7.

18. Slootweg PJ, Mueller H. Differential diagnosis of fibro-osseous jaw lesion: a histological investigation on 30 cases. J Craniomaxillofac Surg 1990 Jul; 18(5): 210-4.

19. White SC, Gooz PW. Oral radiology principles and interpretation. 4th ed. St Louis: Mosby, 2000.

20. Zhang J, Wang H, He X, Niu Y and Li X. Radiographic examination of 41 cases of odontogenic myxomas on the basis of conventional radiographs. Dentomaxillofac Radiol 2007 Mar; 36(3):160-7.

Recebido em: 19/10/2007 Aceito em: 8/12/2007 